Original Article

\title{
PRE AND POST EXPOSURE ANALYSIS OF CHLORHEXIDINE GLUCONATE'S EFFECTIVENESS AGAINST BACTERIAL CONTAMINATION OF STETHOSCOPES
}

\author{
AISHWARYA HARIKRISHNAN ${ }^{1}$, G. BHUVANESHWARI ${ }^{2}$ \\ ${ }^{1}$ Saveetha Medical College and Hospital, Chennai, ${ }^{2 D e p a r t m e n t ~ o f ~ M i c r o b i o l o g y, ~ S a v e e t h a ~ M e d i c a l ~ C o l l e g e ~ a n d ~ H o s p i t a l, ~ C h e n n a i ~}$ \\ Email: aishwarya.h2000@gmail.com
}

Received: 16 Sep 2020, Revised and Accepted: 17 Nov 2020

\section{ABSTRACT}

Objective: The present study is to assess the effectiveness of chlorhexidinegluconate as a disinfectant for the stethoscope diaphragm, to survey the routine practices followed by health care professionals (HCPs) towards stethoscope disinfection and to ascertain their general awareness on the matter.

Methods: Questionnaire-based study conducted to assess the degree of awareness and attitude of HCPs towards stethoscope disinfection procedures. 200 responses were obtained and analyzed. Randomized, blind trial comparing bacterial load of stethoscope post-exposure to chlorhexidinegluconate vs. ethyl alcohol. The swabs collected from stethoscope diaphragm before and after exposure to disinfectant, were cultured using spread plate technique on nutrient agar. CFU count was compared.

Results: Out of 200, nearly all HCPs part of this study were aware that stethoscopes could be linked to transmission of healthcare-associated infections (HCAIs) but only 78.5\% (157) were accustomed to stethoscope disinfection practices. Although only 13.5\% (27/200) of participants believed that chlorhexidinegluconate was effective, this study found it to be highly efficacious against bacterial recontamination.

Conclusion: We stress upon the importance of regular disinfection of the stethoscope after each use and encourage frequent cleaning of the device with chlorhexidinegluconate, which may reduce bacterial recontamination for a longer period.

Keywords: Effectiveness, Chlorhexidinegluconate, Stethoscope, Disinfection, Healthcare-associated infection, Nosocomial, Healthcare professional, Physician, Questionnaire

(C) 2021 The Authors. Published by Innovare Academic Sciences Pvt Ltd. This is an open access article under the CC BY license (https://creativecommons.org/licenses/by/4.0/) DOI: https://dx.doi.org/10.22159/ijcpr.2021v13i1.40799. Journal homepage: https://innovareacademics.in/journals/index.php/ijcpr

\section{INTRODUCTION}

The stethoscope is the medical device standardly used by health care workers for auscultation of a patient during clinical examination. As the diaphragm of the physician's stethoscope is the portion of the instrument which makes contact with large numbers of infected patients throughout the day, it is prone to becoming colonized by microbial organisms. Evidence has shown that the diaphragms and bells of stethoscopes randomly sampled in a health care setting, such as a hospital, are almost universally contaminated by potential nosocomial pathogens, most often by Staphylococci, Clostridium difficile, resistant gram-negative bacilli, and even viruses [1]. Healthcare workers (HCWs) often overlook non-critical healthcare tools including stethoscopes and sphygmomanometers as sources of infection. Studies have, however, demonstrated a significant risk of transmission of Healthcare-Associated Infections (HCAIs) with these devices [2].To avoid patient to patient transmission of communicable infections, the introduction of disposable stethoscopes kept at the bedside of each patient has even been considered [3]. But this does not seem to be a practical solution, especially in the numerous smaller, limited-resource medical setups across India. Hence, disinfectant precautions remain the simplest, most feasible approach to avoid cross-contamination of HCAIs during medical practice, provided there is physician compliance to aseptic precautions. The effectiveness of disinfection can vary with the composition of the disinfectant as well as with the frequency of disinfection. Chlorhexidinegluconate was opted for over alcoholic agents (such as ethyl alcohol). Although disinfection with alcohol is known to decrease the bacterial load on stethoscopes, they often become recontaminated quickly because alcohol evaporates and its effect is extended only if items remain immersed in it [4]. Thus, the present study is to assess the effectiveness of chlorhexidinegluconate as a disinfectant for the stethoscope diaphragm, to survey the routine practices followed by health care professionals towards stethoscope disinfection and to ascertain their general awareness on the matter.

\section{MATERIALS AND METHODS}

\section{Part A: Questionnaire-based study}

This cross-sectional study was conducted between June 2020 and August 2020 in Saveetha Medical College and Hospital, a tertiary care hospital in Chennai, Tamil Nadu, India. The degree of awareness and attitude of health care workers (HCWs) towards stethoscope disinfection procedures was assessed through a questionnaire-based study. The questionnaire was prepared using Google Forms for ease of circulation and to ensure that safe distancing was maintained during the COVID-19 pandemic. 200 health care professionals (physicians, surgeons, nurses, medical interns included) took part in the survey and the data received was subsequently analyzed.

\section{Exclusion criteria}

Medical personnel who reported to very infrequent stethoscope use as well as medical students belonging to their pre-clinical years of study were excluded from the study population.

\section{Part B: Microbiological analysis}

After obtaining written informed consent from each participant, the stethoscopes of those willing were collected. Before exposure to any disinfectant agents, sterile swabs were taken from the chest piece of each stethoscope in compliance with standard laboratory protocol. The microbiological sample (of volume $0.1 \mathrm{ml}$ ) was inoculated using spread plate technique[5] and the bacterial growth was quantitatively analyzed in terms of $\mathrm{CFU} / \mathrm{ml}$ (number of colony forming units per milliliter).

The stethoscopes were blindly assigned into 2 test groups of 5 with Chlorhexidinegluconate and ethyl alcohol being applied onto the diaphragms of stethoscopes of group A and group B respectively. Further samples were collected after one minute and after fifteen minutes of exposure to chlorhexidinegluconate or ethyl alcohol from the same device. The growth of bacterial colonies on the agar plate was 
noted in samples obtained from Group A and Group B stethoscopes and the number of $\mathrm{CFU} / \mathrm{ml}$ seen pre-exposure vs. post-exposure to the respective disinfectants was compared and contrasted.

\section{RESULTS AND DISCUSSION}

Out of the 200 health care professionals (HCPs) who took part in the questionnaire-based study, only $71 \%(142 / 200)$ of participants had their own stethoscope for daily practice. $20.5 \%(41 / 200)$ stated that they made use of the stethoscope common to that ward, while $11 \%$ $(22 / 200)$ preferred to utilize the stethoscope kept at the bedside of each patient.

Only $78.5 \%(157 / 200)$ of HCPs had the practice of disinfecting their stethoscopes, of which $64 \%(128 / 200)$ admitted to applying handrub or sterilium to clean the device, whereas $23 \%(46 / 200)$ made use of anti-bacterial wipes and 6.5\% (13/200) used alcoholic gels.

When asked what were the reasons due to which a HCP would fail to clean their stethoscope on any given occasion, most responses pointed towards being pressed for time during clinical practice, ranging from 'forgetting to do so'-39\% (78/200) to 'lack of time'$28 \%(56 / 200)$, 'using a shared stethoscope'-10.5\% (21/200) and 'lack of access to disinfectants'-5\% (10/200). Surprisingly, 9\% of respondents $(18 / 200)$ expressed their concerns that application of disinfectants on their stethoscope would damage the device and hence, refrained from using any disinfectant agents at all. However, the increased risk of transmission of HCAIs seems a far greater threat than the possibility of damage to the stethoscope, a claim which lacks proper research-based evidence. Meanwhile, the role of medical devices as a source of infection is already well-established [6].Hence, it can be insisted upon that stethoscopes and all other medical devices closely associated with patient care, are cleaned regularly after each use as recommended by the CDC guidelines[7,8].

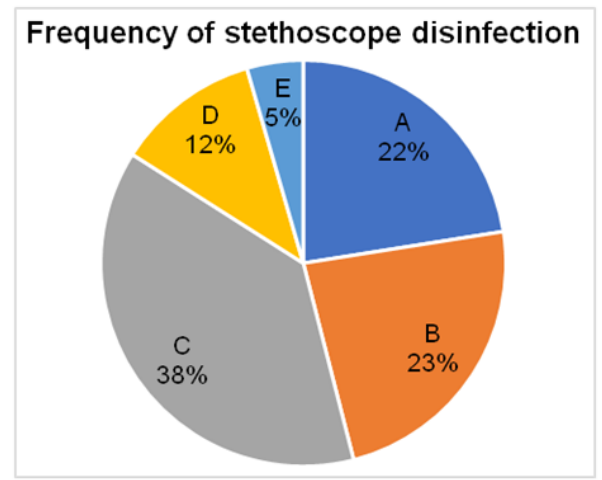

Fig.1: Frequency of stethoscope disinfection (A-Once a day, BBefore and after a day of practice, C-after each patient, D-Not at all cleaned, E-Only once a week)
In contrast to this study, C. Saunders et al. reports a higher proportion $(21.4 \%)$ of respondents having never disinfected their stethoscopes before and a much lower proportion (9.7\%) of participants with the habit of cleaning at least once daily [9] As disinfection of non-critical health care tools like the stethoscope are often overlooked when setting the hospital standard guidelines for infection control [10], we urge more medical setups to emphasize upon stethoscope disinfection protocols. This could have a positive effect in increasing the frequency of cleaning and as well as reminding HCPs of the necessity of such simple but effective hygiene measures.

$62 \%(124 / 200)$ of HCPs were under the impression that, the part of the stethoscope with the greatest risk of becoming colonized by microorganisms, was the diaphragm, $16.5 \%(33 / 200)$-the earpiece and $16.5 \%(33 / 200)$-the bell. This appeared to directly influence which portion of the stethoscope each HCW preferred to clean (fig. 2).

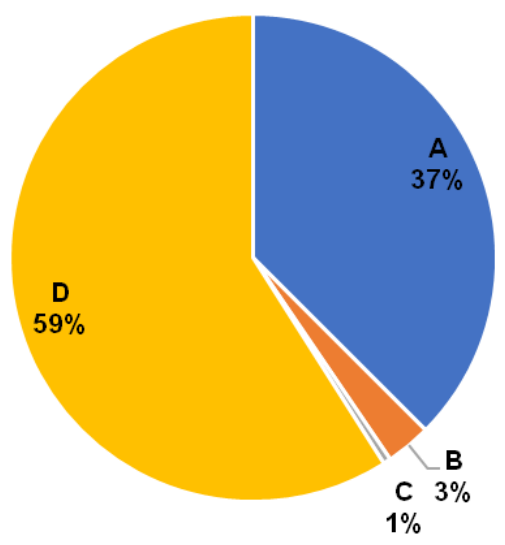

Fig.2: Portion of the stethoscope routinely cleaned by the HCW (A-Bell and diaphragm, B-Stethoscope earpieces, C-Tubing, DAll parts including diaphragm, bell, earpieces and tubing)

$70 \%$ isopropyl alcohol was believed to be the most effective agent against recontamination by $75.5 \%(151 / 200)$ of all participants of the study, whilst the disinfectant chlorhexidinegluconate was only considered to be effective by $13.5 \%(27 / 200)$ of participants. However, on conducting pre-and post-exposure culture and analysis of the samples obtained from the stethoscope diaphragm of health care professionals, it was found that chlorhexidinegluconate was also a highly effective surface disinfectant (fig. 3). Even previous literature supports our findings and reiterates that Chlorhexidinegluconate is an efficacious disinfectant agent, especially against non-fermenting Gram negative bacilli (NFGNB) which happen to be a common culprit of HCAIs [11].

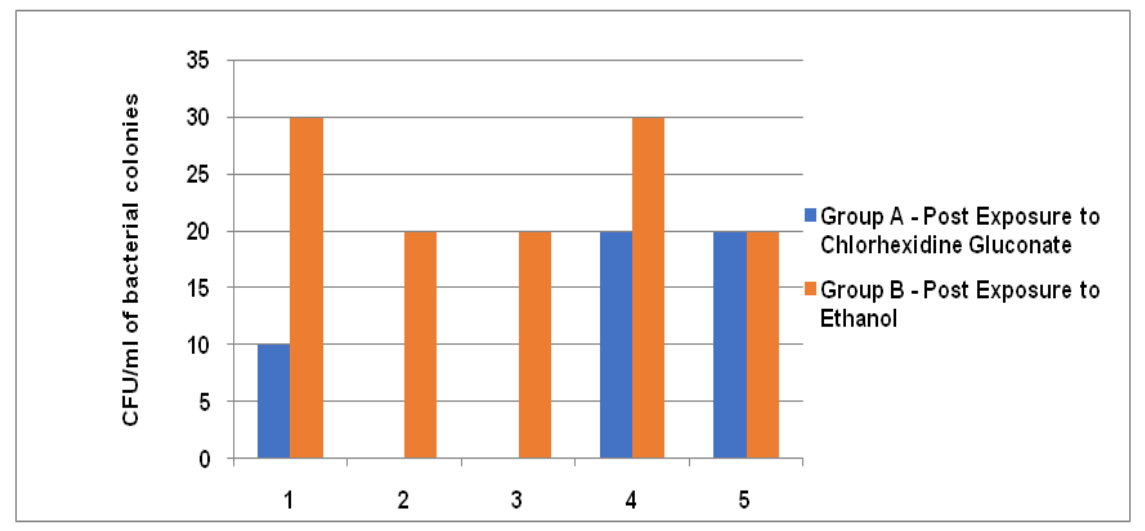

Fig.3: Post exposure of group a (Chlorhexidinegluconate) vs group B (Ethanol) 


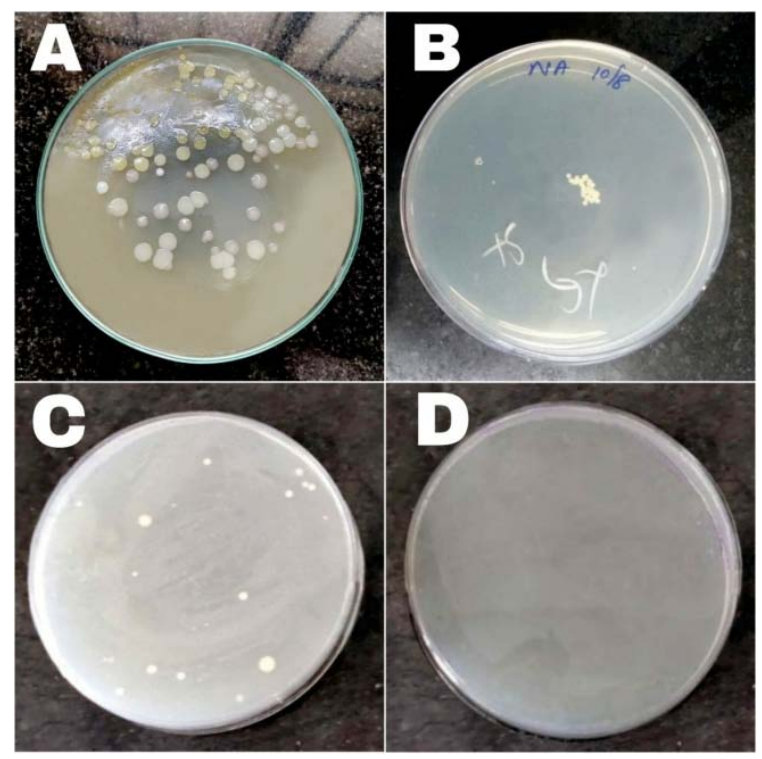

Fig.4: Bacterial culture using spread plate technique (A and C-Pre exposure to disinfectant, B-Post exposure to ethyl alcohol, D-Post exposure to chlorhexidine)

Table 1: Group A-pre vs. post exposure to chlorhexidinegluconate

\begin{tabular}{lll}
\hline S. No. & Pre-exposure to chlorhexidinegluconate (CFU/ml) & Post-exposure to chlorhexidinegluconate (CFU/ml) \\
\hline 1 & 250 & 10 \\
2 & 120 & 0 \\
3 & 80 & 0 \\
4 & 160 & 20 \\
5 & 180 & 20 \\
\hline
\end{tabular}

Table 2: Group B-pre vs post exposure to ethyl alcohol wipes

\begin{tabular}{lll}
\hline S. No. & Pre-exposure to ethyl alcohol wipes (CFU/ml) & Post-exposure to ethyl alcohol wipes (CFU/ml) \\
\hline 1 & 350 & 30 \\
2 & 150 & 40 \\
3 & 250 & 20 \\
4 & 120 & 30 \\
5 & 160 & 20 \\
\hline
\end{tabular}

Majority (91\% or $183 / 200$ ) of the health care professionals agreed that medical instruments could be the source of potentially pathogenic microorganisms but only $31.5 \%(63 / 200)$ correctly estimated the incidence of HCAIs amongst patients of hospitals in India, around the time of the study. Many HCWs severely underestimated the incidence of HCAIs prevalent in urban medical setups across India, which once again contributes to the attitude of the physician towards aseptic precautions in routine practice and his/her strict adherence to them. In concordance with this study, a 2018 American survey concluded that nearly all physicians believed that stethoscopes could harbour potential pathogens implicated in HCAIs. But there was a general lack of consensus on which disinfectant was most efficacious or what the optimal frequency of disinfection might be [12].

\section{CONCLUSION}

Although nearly all healthcare professionals part of this study were aware that stethoscopes could be linked to transmission of HCAIs, only around three quarters of them were accustomed to stethoscope disinfection practices. A wide range of disinfectants were utilized but majority of HCPs believed only 70\% isopropyl alcohol to be most effective. This study found chlorhexidinegluconate to also be highly efficacious against bacterial contamination on the stethoscope and would recommend regular disinfection of the device after each use. The implications of bacterial contamination of the stethoscope and its involvement in HCAIs are a matter of public health concern. Thus, disinfecting the device with chlorhexidinegluconate may help in reducing bacterial contamination for a longer period.

\section{ACKNOWLEDGEMENT}

To the 200 health care professionals who responded completely to the questionnaire that was circulated, thank you for sparing your time and contributing towards this study. Special mentions to KaviyaGopal and AnandVishwesh Mani for their valuable inputs regarding statistical analysis, and to my friends and family for their help in completing this work.

\section{FUNDING}

Nil

\section{AUTHORS CONTRIBUTIONS}

All the authors have contributed equally.

\section{CONFLICTS OF INTERESTS}

Declared to none

\section{REFERENCES}

1. Maki DG. Stethoscopes and health care-associated infection. Mayo ClinProc March 2014;89:277-80.

2. Weldegebreal F, Admassu D, Meaza D. Non-critical healthcare tools as a potential source of healthcare-acquired bacterial infections in 
eastern Ethiopia: a hospital-based cross-sectional study. SAGE Open Med 2019;7.DOI:10.1177/2050312118822627.

3. Lecat P. A "solution" for infectious stethoscopes? Mayo ClinProc 2014;89:1318-20.

4. Alvarez JA, Ruiz SR, Mosqueda JL. Decontamination of stethoscope membranes with chlorhexidine: should it be recommended? Am J Infect Control 2016;44:e205-e209.

5. Sanders ER. Aseptic laboratory techniques: plating methods. J Vis Exp 2012;63:e3064.

6. HA Bukharie, H Al-Zahrani, AMRubaish. Bacterial contamination of stethoscopes. J Family Community Med 2004;11:31-3.

7. A Campos Murguia, X Leon Lara, JM Munoz. Stethoscopes as potential intrahospital carriers of pathogenic microorganisms. Am J Infect Control 2014;42:82-3.
8. Jain A, Shah H, Jain A, Sharma M. Disinfection of stethoscopes: gap between knowledge and practice in an Indian tertiary care hospital. Ann Trop Med Public Health 2013;6:236-9.

9. C Saunders. Factors influencing stethoscope cleanliness among clinical medical students. J Hospital Infection 2013;84:242-4

10. Pratiksha S, Atosh T, Priti A. The role of stethoscopes in the transmission of hospital infections. Int J CurrMicrobiolApplSci 2019;8:2472-8.

11. GBhuvaneshwari, AS Shameembanu, M Kalyani. Disinfectant susceptibility testing of non-fermenting gram negative bacilli. Res J Pharm Tech 2018;11:1313-5.

12. GW Ghumman, N Ahmad, A Pop-Vicas. Stethoscope cleaning during patient care. RI Med J 2018. p. 18-20. 